\title{
Expression and phylogeny of multidrug resistance protein 2 and 4 in African white backed vulture (Gyps africanus)
}

\author{
Bono Nethathe ${ }^{\text {Corresp., 1, } 2}$, Aron Abera ${ }^{3}$, Vinny Naidoo ${ }^{2}$ \\ ${ }^{1}$ Department of Food Science and Technology, University of Venda, Thohoyandou, Limpopo, South Africa \\ 2 Department of Paraclinical Science, University of Pretoria, Onderstepoort, Pretoria, South Africa \\ 3 Inqaba Biotechnology, Sunnyside, Pretoria, South Africa \\ Corresponding Author: Bono Nethathe \\ Email address: bono.nethathe@univen.ac.za
}

Diclofenac toxicity in old world vultures is well described in the literature by both the severity of the toxicity induced and the speed of death. While the mechanism of toxicity remains unknown at present, the necropsy signs of gout suggests primary renal involvement at the level of the uric acid excretory pathways. From information in the chicken and man, uric acid excretion is known to be a complex process that involves a combination of glomerular filtration and active tubular excretion. For the proximal convoluted tubules excretion occurs as a two-step process with the basolateral cell membrane using the organic anion transporters and the apical membrane using the multidrug resistant protein to transport uric acid from the blood into the tubular fluid. With uric acid excretion seemingly inhibited by diclofenac, it becomes important to characterize these transporter mechanism at the species level. With no information being available on the molecular characterization/expression of MRPs of Gyps africanus, for this study we used next generation sequencing, and Sanger sequencing on the renal tissue of African white backed vulture (AWB), as the first step to establish if the MRPs gene are expressed in AWB. In silico analysis was conducted using different software to ascertain the function of the latter genes. The sequencing results revealed that the MRP2 and 4 are expressed in AWB vultures. Phylogeny of avian MRPs genes confirms that vultures and eagles are closely related, which could be attributed to having the same ancestral genes and foraging behavior. In silico analysis confirmed the transcribed proteins would transports anionic compounds and glucose. 
1 Expression and phylogeny of multidrug resistance protein 2 and 4 in African White Backed

2 vulture (Gyps africanus).

3 Bono Nethathe ${ }^{1,3}$, Aron Abera ${ }^{2}$, Vinny Naidoo ${ }^{1}$

4 1Department of Paraclinical Science, University of Pretoria, Onderstepoort, Pretoria, South

5 Africa.

6 Inqaba Biotechnology, Pretoria, South Africa.

7 3 Department of Food Science and Technology, University of Venda, Thohoyandou, Limpopo,

8 South Africa.

9 Corresponding Author:

10 Bono Nethathe ${ }^{1}$

11 University road, Thohoyandou, Limpopo, 0950, South Africa

12 Email address: bono.nethathe@univen.ac.za

\section{Abstract}

Diclofenac toxicity in old world vultures is well described in the literature by both the severity of the toxicity induced and the speed of death. While the mechanism of toxicity remains unknown at present, the necropsy signs of gout suggests primary renal involvement at the level of the uric acid excretory pathways. From information in the chicken and man, uric acid excretion is known to be a complex process that involves a combination of glomerular filtration and active tubular excretion. For the proximal convoluted tubules excretion occurs as a two-step process with the basolateral cell membrane using the organic anion transporters and the apical membrane using the multidrug resistant protein to transport uric acid from the blood into the tubular fluid. With uric acid excretion seemingly inhibited by diclofenac, it becomes important to characterize these transporter mechanism at the species level. With no information being available on the molecular characterization/expression of MRPs of Gyps africanus, for this study we used next generation sequencing, and Sanger sequencing on the renal tissue of African white backed vulture (AWB), as the first step to establish if the MRPs gene are expressed in AWB. In silico analysis was conducted using different software to ascertain the function of the latter genes. The sequencing results revealed that the MRP2 and 4 are expressed in AWB vultures. Phylogeny of avian MRPs genes confirms that vultures and eagles are closely related, which could be attributed to having the same ancestral genes and foraging behavior. In silico analysis confirmed the transcribed proteins would transports anionic compounds and glucose. 


\section{Introduction.}

In India, birds belonging to the Gyps genus (Oriental white-backed vulture, Gyps bengalensis; long-billed vulture, G. indicus and slender-billed vulture, G.tenuirostris) were in grave danger of extinction in the 1990s following their inadvertent exposure to veterinary diclofenac that found its way into their food chain (Oaks et al., 2004). Following their single exposure to quantities of drug that equated to a dose in the region of $0.8 \mathrm{mg} / \mathrm{kg}$, birds were found dead within 48 hours of said exposure with signs of severe visceral gout and associated renal and hepatic damage (Oaks et al., 2004, Green et al., 2004; Shultz et al., 2004; Swan et al., 2006). Despite the diclofenac induced deaths being largely recognized as one of the worst environment intoxications in recent times, the mechanism behind the evident gout is still incompletely understood. One of the theories put forward, suggests that toxicity is due to the inhibition of the uric acid transporter channels in the renal tubular epithelial cells (Naidoo et al., 2007), which would be consistent with diclofenac inhibition of uric acid transport in mammals (Khamdang et al., 2002; Nozaki et al., 2007; Burckhardt, 2012).

The Multidrug resistance proteins (MRPs) are part of the uric acid transporters that play an important role in the excretion of uric acid from the intracellular environment, following their movement therein by the organic anionic transporters in mammals (Sweet, 2005, 2010; Van wert et al., 2010). The MRPs family also known as ATP-binding cassette subfamily C (ABCC) was first described as a drug resistance protein by Cole et al. (1992) when they managed to clone the MRP1 gene in human (Cole et al., 1992). They first associated it with transporting anti-cancer drugs (Haimeur et al., 2004). MRP1 is generally regarded as the godfather of the family and further research has since described five homologs named MRP2-6 (Kool et al., 1997, 1998, 1999).

In general, the MRP protein functions as an extrusion pump system responsible for moving substances from within the cell into the extracellular environment e.g. in biliary transport it moves substances into the biliary tract and bile (Taniguchi et al., 1996; Kool et al., 1997), while MRP2 expressed in the proximal renal tubule endothelial cells found in apical membrane plays a role in the excretion of small intracellular organic anions (Sekine et al., 2006). Examples of substance transported by renal MRP2 are lipophilic substances conjugated to glutathione, glucuronate, or sulfate (König et al., 1999). The MRP transport proteins are distributed throughout the body with MRP3 and MRP4 found in the pancreas, bladder, gut, lung, prostate, ovary, muscle, testis, kidney and gallbladder respectively while MRP5 being ubiquitous (Kool et al., 1997, 1999; Lee et al., 1998; Kiuchi et al., 1998; KÖnig et al., 1999). Nonetheless, the main MRPs transporters are located in the liver and kidney.

With diclofenac having the ability to induce severe hyperuricemia and gout in the vultures (Naidoo and Swan, 2009), this study focuses on characterizing the last step in uric acid transport. More specifically, we focused on the molecular characterizing of the MRP2 and MRP4 
transporter in the kidney of Gyps africanus (African white backed) vulture. In addition to being known to transport organic anions including uric acid, it is believed that these channels function in the same manner as in mammals. The rationale for this approach, it that once the channel has been characterized, more specific expression and cloning studies can be undertaken on these channels. While no such studies have been undertaken in an avian species, studies on cloned MRP transporter proteins from humans have been successfully used to evaluate the effect of specific non-steroidal anti-inflammatory drugs as inhibitors of these transporters (El-Sheikh et al., 2007). Moreover we compare the results to the recent study by Barik et al. (2019) which characterized MRP4 gene on Gyps Himalyanesis for comparison to results with obtained in this study (Barik et al., 2019).

\section{Material and methods.}

\subsection{Sample collection}

Before the commencement of experiments, ethical clearance for collection of samples was conducted according to the guidelines approved by University of Pretoria Animal Ethics committee (V108-16). Two African white-backed (AWB) (Gyps africanus) vultures of unknown age with a badly fractured wing and had not been treated with any analgesic previously were euthanized at Faculty of Veterinary Science, University of Pretoria. The cranial lobe part of the kidneys was harvested immediately after euthanasia and stored in cryogenic vials containing RNAlater (Whitehead Scientific, Cape Town, South Africa) and stored at $-80^{\circ} \mathrm{C}$ freezer until analysed.

\subsection{Next generation sequencing (NGS)}

Total RNA was extracted from AWB1`s kidney using the RNeasy plus mini kit (Qiagen) according to the manufacturer's instructions and transported to Agricultural Research Council (ARC, Onderstepoort, Pretoria, South Africa) on dry ice for sequencing. DNA contamination was removed from total RNA using DNase. The RNA fragments were reverse transcribed into cDNA and sequencing adaptors were ligated. Finally, the ends of the cDNAs were sequenced using Illuminia Truseq mRNA stranded Ran preparation kit on Hiseq 2500 v4 $2 \times 125 \mathrm{bp}$ chemistry model. The result obtained from sequencing was 50 million short reads which were 125 nucleotides long each. Prior assembly of the raw reads, several quality control steps were conducted on the raw reads using the online Galaxy platform (Blankenberg et al., 2010) and fast quality check (FASTQC) (Andrews, 2014) to check overall quality of the sequences, duplication and adapters. After checking the quality of the sequences, the adapters and PCR duplicates were removed using the programme Trimmomatic (Bolger et al., 2014). The pre-processed reads were assembled into transcripts using TRINITY (Grabherr et al., 2011). The assembled transcriptome was converted to a local blast database NCBI (Altschul et al., 1990) and sequences were identified based on the predicted MRP2 (XM_030031380.1) and MRP4

(XM_030036470.1) sequences from the Golden eagle genome. The Golden Eagle was selected 
112 as another study completed in our laboratory has shown them to be closely related species as

113 well as having both channels present. Transcript quantification was undertaken using Sailfish

114 with reference transcripts from the de novo analysis. Alignment of AWB MRP4 and Gyps

115 Himalyanesis was also conducted using Clusta omega to reveal similarity.

116

117

118

119

120

121

122

123

124

125

126

127

128

129

130

131

132

133

134

135

136

137

138

139

140

141

142

143

144

145

146

147

148

2.3. Confirmation of transcriptome MRP2 and MRP4 using Sanger sequencing.

Fresh total RNA was extracted from AWB2`s kidney using Quick RNA Miniprep kit following instructions from the manufacturer (Zymo Research, USA). cDNA synthesis was performed using Lunascript RT supermix kit from New England Biolabs (NEB), (USA) according to the manufacturer's instructions. Primers of MRP2 and MRP4 were designed based on the transcriptome sequences generated from illuminia next generation sequencing. The special primers were synthesized at Inqaba Biotechnology, Pretoria, South Africa. Using the latter set of primers, PCR was performed using One taq polymerase kit from NEB and AWB`s kidney cDNA as a template. The amplification protocol for all the targets was as follows: Initial denature $94^{\circ} \mathrm{C}$ $30 \mathrm{sec}, 40$ cycles of (denature $94^{\circ} \mathrm{C}$ for $30 \mathrm{sec}$; annealing $55^{\circ} \mathrm{C}$ for $30 \mathrm{sec}$ and elongation $68^{\circ} \mathrm{C}$ for $2 \mathrm{~min}$ ) and final elongation $68^{\circ} \mathrm{C} 5 \mathrm{~min}$. However for MRP2 segment 1 , the former did not work so the following cycling conditions where used, Initial denature $98^{\circ} \mathrm{C} 30 \mathrm{sec}, 40$ cycles of (denature $98^{\circ} \mathrm{C}$ for $30 \mathrm{sec}$; annealing $65^{\circ} \mathrm{C}$ for $30 \mathrm{sec}$ and elongation $72^{\circ} \mathrm{C}$ for $2 \mathrm{~min}$ ) and final elongation $72^{\circ} \mathrm{C} 2 \mathrm{~min}$. The PCR products was run on $1 \%$ agarose gel. The gel was viewed for desired bands using gel documentation system and PCR products of the desired bands was further cleaned up using Exopsup from NEB and sequences were generated using ABI 3500X1 genetic analyser.

\section{$\underline{\text { 2.4. Phylogenetic analyses }}$}

After confirming the NGS data with Sanger sequencing, searches for homologous sequences were performed using BLASTn (Altschul et al., 1990). Thirty-five (MRP2) and 36 (MRP4) genes that were $85 \%$ and above identical to NGS sequences were downloaded from GenBank (NCBI). A multiple sequence alignment was performed along with the downloaded sequences, using muscle algorithm (Molecular Evolutionary Genetics Analysis version X (MEGA X)) software package (Edgar, 2004; Kumar et al., 2018). The evolutionary history was inferred by using the Maximum Likelihood method and Tamura-Nei model for MRP2 (Tamura and Nei, 1993) while MRP4 was Maximum Likelihood method and General Time Reversible model (Nei and Kumar, 2000). The bootstrap consensus tree inferred from 1000 replicates was taken to represent the evolutionary history of the taxa analyzed (Felsenstein, 1985). Branches corresponding to partitions reproduced in less than 50\% bootstrap replicates were collapsed. The percentage of replicate trees in which the associated taxa clustered together in the bootstrap test (1000 replicates) are shown next to the branches (Felsenstein, 1985). Evolutionary analyses were performed using MEGA X (Kumar et al., 2018). 


\section{$\underline{\text { 2.5. In silico analysis }}$}

The obtained MRP2 and MRP4 NGS sequences were converted in Expasy (Gasteiger et al., 2003 ) to protein sequences for their open reading frame. The deduced amino acids sequences were analysed by following software; Transmembrane helix prediction using a Markov model (Sonnhammer et al., 1998), Swiss model Protparam for functional prediction of the protein (De Castro et al., 2006) and PHYRE2 (Kelly et al., 2015) for prediction of 2-D and 3-D structures. PROTTER sequence database was used to predict N-glycosylation sites (Omasits et al., 2013) and Expasy database was also used to examine other possible post-glycosylation and phosphorylation. The Walker A, ABC signature motif and Walker B segments which are hallmarks of human MRP 2 and 4 were evaluated for its presence (Hipfner et al., 1999; Choudhuri and Klaassen 2006), to conclude that the proteins were functional MRP. This was compared for both nucleotide binding domain 1\&2 (Ren et al., 2004).

\section{$\underline{\text { 3. Results. }}$}

\subsection{Next generation sequencing.}

The assembled transcriptomes was compared to the Golden Eagle MRP2 (XM_030031380.1) and MRP4 (XM_030036470.1) sequences. The predicted AWB MRP2 (MN691108) and MRP4 (MN691109) genes were submitted to NCBI and consisted of 5212 and $4061 \mathrm{bp}$ respectively. The MRP2 and 4 alignment of the AWB vulture and the golden eagle were 98.20\% and 99.16\% similarity respectively. Oddly the AWB and Gyps Himalyanesis (KX168697.1) alignment of MRP4 gene revealed $94.06 \%$ similarity.

\subsection{Sanger sequencing of data.}

To confirm the above NGS results, Sanger sequencing was conducted. The cDNA from the AWB kidney was used as template. MRP2 was amplified into two segments using primers design based on NGS data. The PCR products were viewed on a gel under UV documentation and it revealed product size of 2754bp and 2550bp for segment 1 and 2 respectively (Supplementary Figure 1). Moreover amplification of MRP4 was also divided into two segments and PCR products were 2241bp and 2136bp for segment 1 and 2 in that order. The PCR products were sequenced and the sequences were analysed for consensus which revealed $4537 \mathrm{bp}$ for MRP2 and 3897bp for MRP4. While full coverage was not possible, based on the degree of coverage and similarity between NGS and Sanger of $99.76 \%$ and $99.43 \%$ for MRP2 and MRP4 respectively was observed. The similarity gave us confidence that the NGS sequences are a true representative of the MRPs sequences. These sequences could not deposited in the NCBI as a result of stop codons within the sequences, which we believe were sequencing errors. 
185

186

187

188

189

190

191

192

193

194

195

196

197

198

199

200

201

202

203

204

205

206

207

208

209

210

211

\subsection{Phylogenetic analysis.}

The AWB predicted genes were further analysed to confirm its homogeneity compared to the MRP gene of other avian species. The phylogenetic tree topology revealed 2 distinct clades for the vulture and chicken with the golden eagle, bald eagle and AWB vulture belonging to the same clade. The percentage similarity between vulture, eagles and chicken is stated on the internal nodes numbers representing the percentage of 1000 replicates for which the same branching patterns were attained. (Fig 1 and 2).

\subsection{In silico predictions.}

The predicted AWB MRP2 sequence generated open reading frame (ORF) containing 1552 amino acids while MRP4 ORF contained 1287 amino acids with a predicted molecular weight 173610.3 and 145113.90 Daltons respectively. Scan prosite results revealed that the above proteins are likely ABC transporter integral membrane type 1 fused domain (ABC-TMF1) and ABC transporter 2. Secondary structure prediction with PHYRE2 revealed that the predicted MRP2 amino acids contain $62 \%$ of alpha-helix, $7 \%$ of beta- strand and $30 \%$ transmembrane helix. While MRP4 PHYRE2 results showed the former to contain $61 \%$ alpha-helix, 9\% of betahelix and 26 to be coiled in nature. TMHMM software analysis for MRP2 revealed the presence of two nucleotide binding domains (NBDs) and two transmembrane domains (TMDs) consisting of 6 and 5 transmembrane helices $(\mathrm{TMH})$ respectively with an extra transmembrane domain (TMD) with $5 \mathrm{TMH}$ and for MRP4 it revealed their presence of two NBDs and two TMDs containing 5 and $6 \mathrm{TMH}$ each. PROTTER sequence database was used for prediction of $\mathrm{N}$ glycosylation sites for MRP2 and were found at position 15, 106, 284, 551, 620, 725, 773, 886, 1019, 1193, 1254, 1383, 1443 and MRP4 sites were at position 428, 706, 716, 1009, 1138 for both AWB vulture and golden eagle moreover confirming the above results (Figure 3 ). The quaternary structures predicted by PHYRE2 from MRPs amino acids revealed the presence of two NBDs for both MRPs protein (Figure 3). The Walker sequence and ABC signature motif are presented in Figure 4. For both MRP 2 and 4, these sequences were present with one amino acid difference, when a difference was present, in comparison to human MRP2\&4. 
212

213

214

215

216

217

218

219

220

221

222

223

224

225

226

227

228

229

230

231

232

233

234

235

236

237

238

239

240

241

242

243

244

245

246

247

248

\section{Discussion.}

Previous studies revealed that the Gyps species found dead in India was due to the presence of residues of diclofenac in the carcass they fed upon (Oaks et al., 2004), which caused signs of abnormally high level of plasma uric acid prior to death (Naidoo and Swan 2009). With uric acid excretion primarily occurring in the kidneys, it has been speculated that diclofenac inhibited uric acid excretion within the renal tubules. The one shortcoming of this hypothesis is that the mechanisms of uric acid excretion is not yet completely understood in vultures. Thus to understand the mechanism of toxicity of diclofenac, the mechanism of uric acid excretion needs to be elucidated.

From studies in the chicken, it is known that uric acid in the plasma is excreted by the renal tubules by the organic anion transporters which transports uric acid from the plasma into the cell; while subsequent transport from within cell into the renal tubule being mediated by the MRP transporters (Sweet, 2005, 2010; Van wert et al., 2010). For this study we focused on the MRP2 and 4 transporters, since Haritova et al. (2010) and Bataille et al. (2008), were respectively able to demonstrate the presence of MRP2 and MRP4 in the chicken kidney. More specifically, we used the AWBV as our test species, which as a vulture species was the second species demonstrated to be highly sensitive to the toxic effects of diclofenac, and more so under controlled laboratory conditions by Swan et al (2006). In a later rebuttal, Cuthbert et al. (2007) indicated that a sample size of two birds was sufficient to demonstrate toxicity due to the reported $\mathrm{LD}_{50}$ in other vulture species. Also in a subsequent study, when the plasma diclofenac plasma concentration was subjected to non-compartmental analysis by Naidoo et al (2009), they were able to demonstrate that toxicity in the study vulture species was associated with a very long half-life of 11 and $22 \mathrm{~h}$ in the two birds.

For this study both the study methods (NGS and Sanger sequencing) were able to demonstrate that the MRP2 and MRP4 transporters were expressed in the AWB vulture's kidney. With no primers being described in the study species, that we were aware off, led to our first step of using NGS. NGS as a tool is known to sequence hundreds to thousands of genes at one time without the need for specific primers. The latter method provides the power to discover uncommon or novel variants through deep sequencing (Alekseyev et al., 2018). In order to verify the results obtained from NGS, Sanger sequencing was subsequently carried out since it is the golden standard for sequencing (Sanger, 1975). As for Swan et al. (2006) we also relied on the use of two AWBV. While the sample size is low, the high sensitivity of the species to toxicity suggests a highly conserved mechanism of uric acid excretion. Also, due to the endangered nature of the species under study, it is extremely difficult to obtain fresh tissue for analysis i.e. fresh tissue is only obtainable when birds are euthanised for ethical reasons. This study also relied on in silico analysis to predict the structure and functions of the proteins sequenced as a first step for cloning and functional studies. 
249 In silico analysis with Expasy revealed that the ORFs of MRP2 and MRP4 proteins consisted of

250

251

252

253

254

255

256

257

258

259

260

261

262

263

264

265

266

267

268

269

270

271

272

273

274

275

276

277

278

279

280

281

282

283

284

285

286

287

1552 and 1287 amino acids respectively for both the AWB vulture and golden eagle. This

finding is similar to human MRP2 and MRP4 proteins which comprises of 1545 and 1325 amino acids respectively (Borst et al., 2000). Moreover a recent study by Barik et al., 2019 also revealed that Gyps Himalyanesis MRP4 consisted of 1349 amino acids while Expasy analysis we undertook for the Gallus gallus MRP2 (accession number XM_015288821.1) and MRP4 (accession number NM_001030819) consisted of 1550 and 1330 amino acids respectively. While the similarity in the above protein sequences of MRP2 and MRP4 in size and length maybe revealing that their structures are conserved, the phylogenetic tree only showed a $100 \%$, similarity between vulture and eagle for both MRP2 and MRP4 channels, while the chicken belongs to separate clade. This was in accordance with previous findings by Zhang et al. (2014) who explored macroevolution. In their evaluation using the full genomes of 48 avian species representing all main extant clades, their phylogenetic tree revealed that vulture and eagles share the same clade distinct from the chickens. Jarvis (2014) and his team showed that vultures and eagles also belonged to the same family called accipitrimorphae (Jarvis et al., 2014). The similarity of vulture and eagle is likely attributed to their foraging behaviour as eagles and vultures share a carnivorous diet while chicken is more an omnivore. With uric acid production related to dietary protein concentration and catabolism, it not also surprising that the eagles and vulture would share similar transport mechanism as they would be exposed to the same level of uric acid as an end product of purine metabolism.

In the first step in determining if the evaluated protein was effective, the protein homology results from PHYRE2 were evaluated. For the protein homology, the programme evaluated the similarity of residue probability distributions for each position, secondary structure similarity and the presence or absence of insertions and deletions. For both MRP 2 and 4, the proteins were $57 \%$ and $39 \%$ similar to bovine MRP 1, with near 100\% confidence, supporting the assertion that the channel sequenced where MRP channels. PROTTER and TMHHM results confirmed the two TMDs and NBDs for both proteins. Research on few high-resolution structures of ABC transporters revealed that the former has two TMDs and two NBDs (also known as ABCs) which aligns with our results (Hollenstein et al., 2007; Oldham et al., 2008; Rees et al., 2009). The TMDs and NBDs are important for protein functionality, since MRPs are ATP-binding cassettes (ABC) transporters that require the use of ATP to facilitate the movement of an extensive range of substance within the cells (Linton and Higgins, 1998). In general for the transport, the TMDs binds the transported substrates and NBDs provide the transport energy by binding and hydrolyzing up to two molecules of ATP (Newstead et al., 2009; Khare et al., 2009; Oldham et al., 2007; Ward et al., 2007). Nonetheless some differences have been recorded, with some $\mathrm{ABC}$ s transporter that are not specific transporters with energy also being needed to reorient the TMDs, which is also provided by the NBDs (Jardetzky, 1966; Smith et al., 2002).

Also important in the functionality of the MRP is the presence of the Walker A and B segments which are the ATP binding segments of the NBDs. Comparison of the MRP with their respective

Peer] reviewing PDF | (2020:07:50873:2:0:NEW 29 Oct 2020) 
288 human sequence showed a high degree of similarity for both NBDs. With the single amino acid

289

290

291

292

293

294

295

296

297

298

299

300

301

302

303

304

305

306

307

308

309

310

311

312

313

314

315

316

317

318

319

320

321

322

323

324

325 difference, there appears to be no difference in the ATP binding sequence as the difference was at a variable point as reported by Walker et al. (1982). This leads to the conclusion that the ATP binding sites on the protein was functional. Also of importance is the ABC signature motif. The motif was minimally different to that of the human reported sequence as for the Walker segments. This is an important feature as studies have shown that a mutation in this region renders the transporter unable to function. TMHMM and PROTTER predicted the transmembrane helices and the N-glycosylation sites of the latter proteins with MRP2 having 13 and MRP4 having $5 \mathrm{~N}$-glycosylation sites. The importance of glycosylation for the MRP has been evaluated in other studies. It has been demonstrated that glycosylation is important for the translocation of the MRP from the endoplasmic reticulum to the cell membrane (Zhang et al., 2005). In the absence of glycosylation, the transporter remains inactive. Based on this result, we are confident that the evaluated sequence would result in the expression of a viable drug efflux transporter.

The presence of MRP2 and 4 in the vulture supports the presence of the described uric acid pathways as for other species which provides the first step for the further evaluation of the mechanism of toxicity. The importance of the channels in toxicity is evident from the study by Konig $(1999,2003)$ who showed that rat mutants and humans lacking MRP2 gene had compromised excretion of organic anions from the liver leading to mild liver disease and inherited jaundice (Konig et al., 1999; 2003). This finding was supported by an MRP4 functionality study, where Bakos et al., 1997 and later Ren et al., 2004 demonstrated that the replacement of a highly conserved glycine in NBD1 with an aspartate in vector-transfected cells resulted in intracellular accumulation of the evaluated drugs, confirming their role as efflux transporters. The importance of MRP4 channel was further highlighted in Abcc4-/- mice study, where the absence of the transporter played a role in acute 9-(2-phosphonylmethoxyethyl) adenine (PMEA) toxicity, suggesting a protective role for MRP4 more in the bone marrow, gastrointestinal tract, thymus and spleen (Abla et al., 2008). A similar study on humans comparing two antiviral agents, adefovir (PMEA) and azidothymidine (AZT), by measuring the intracellular accumulation, showed a significantly higher intracellular accumulation of PMEA and AZT in cells without the functional allele in comparison to the controls since these cells were unable to extrude the mentioned drugs (Alba et al., 2008). In addition to the absence of proper expression playing a role in the intracellular accumulation of excretory substrates, the functioning of the MRP2 and MRP4 may be altered by the NSAIDs such as diclofenac. This is best seen by the excretion of prostaglandins by the kidney being inhibited by probenecid, indomethacin, and PAH which all interact with MRP4 (Adachi et al., 2002; Reid et al., 2003). Also in a study by El-Sheikh et al., (2007), the excretion of methotrexate by the MRP 2 and 4 was inhibited by numerous NSAIDs including diclofenac, ketoprofen and phenylbutazone all known to be toxic in vultures. 
326 Despite the importance of the result obtained in this study, it should be noted that this is the first

327

328

329

330

331

332

333

334

335

336

337

338

339

340

341

342

343

344

345

346

347

348

349

350

351

352

353

354

355

356

357

358

359

360

361 step in the process of transporter identification as silico analysis is only able to predict the structure and functions of the proteins sequenced (Punta et al., 2008, Barik et al., 2019). The next step would be to evaluate the functional expression of these transporters. The reason for this is that the sequencing undertaken for this study was only predictive and based on similarity to information published for other species especially man. Also of importance to note is the sample size of two used due to endangered nature of the species evaluated. For the functional studies various technologies may be used. The most commonly available tools would be molecular cloning and transporter studies with specific substrates and inhibitors; immunohistochemistry to identify the location of the transporter in the renal tubules or western blotting to identify protein expression in the kidney (Haimer et al., 2004). These methods do however have disadvantages as with in silico analysis since the assumption is that results are based on conserved physiological function which may not be the case as already evident by the unexpected sensitivity of old world vultures to diclofenac (Klein et al., 1999; Punta et al., 2008; Naidoo et al., 2007). Other concerns with western blotting and immunohistochemistry is that the methodology is reliant on antibodies being able to cross react with vulture tissue which might not be the case since most antibodies are designed for rodent or human use. Molecular cloning, while very effective in studying transporter functionality has the inherent risk of the expressed protein being non-functional due to incorrect folding or improper translocation to the cell membrane e.g. when MRP1 was first cloned it did not overexpress P-glycoprotein, while later it was shown to be overexpressed and well conserved in many multidrug resistance protein family (Mirski et al., 1987; cole et al., 1992; Krishnamachary and center, 1993).

\subsection{Conclusion}

For this study, we show the presence of MRP2 and MRP4 in AWB vulture's kidney, supporting the presence of the expected cellular pathways in uric acid excretion. Phylogenetic analysis also confirmed that vulture and eagle are on the same clade in contrast to chicken moreover inslico revealed two nucleotide binding domains (NBDs) and two transmembrane domains (TMDs) for the latter channels with an extra transmembrane domain (TMD) for MRP4. The former confirm that the transporters function the same as other species. Based on this results, further studies can focus on clonal expression of these transporters to ascertain if they are inhibited by the NSAIDs and thereby explain the hyperuricemia associated with diclofenac toxicity in vultures. 
362

363

364

365

366

367

368

369

370

371

372

373

374

375

376

377

378

379

380

381

382

383

384

385

386

387

388

389

390

391

392

393

394

\subsection{References}

Abla, N., Chinn, L.W., Nakamura, T., Liu, L., Huang, C.C., Johns, S.J., Kawamoto, M., Stryke, D., Taylor, T.R., Ferrin, T.E. and Giacomini, K.M., 2008. The human multidrug resistance protein 4 (MRP4, ABCC4): functional analysis of a highly polymorphic gene. Journal of Pharmacology and Experimental Therapeutics, 325(3), pp.859-868.

Adachi, M., Sampath, J., Lan, L.B., Sun, D., Hargrove, P., Flatley, R., Tatum, A., Edwards, M.Z., Wezeman, M., Matherly, L. and Drake, R., 2002. Expression of MRP4 confers resistance to ganciclovir and compromises bystander cell killing. Journal of biological chemistry, 277(41), pp.38998-39004.

Alekseyev, Y.O., Fazeli, R., Yang, S., Basran, R., Maher, T., Miller, N.S. and Remick, D., 2018. A next-generation sequencing primer-how does it work and what can it do?. Academic pathology, 5, p.2374289518766521.

Altschul, S.F., Gish, W., Miller, W., Myers, E.W. and Lipman, D.J., 1990. Basic local alignment search tool. Journal of molecular biology, 215(3), pp.403-410.

Andrews, S., 2014. FastQC: a quality control tool for high throughput sequence data. Version 0.11. 2. Babraham Institute, Cambridge, UK http:/www. bioinformatics. babraham. ac. $\mathrm{uk} /$ projects/fastqc.

Bakos, É., Klein, I., Welker, E., Szabó, K., Müller, M., Sarkadi, B. and Váradi, A., 1997. Characterization of the human multidrug resistance protein containing mutations in the ATPbinding cassette signature region. Biochemical Journal, 323(3), pp.777-783.

Barik, S., Saini, M., Rana, M. and Gupta, P.K., 2019. Multidrug resistance protein 4 (MRP4) is expressed as transcript variants in both Gallus domesticus and Gyps himalyanesis. Gene, 689, pp.172-182.

Bataille, A.M., Goldmeyer, J. and Renfro, J.L., 2008. Avian renal proximal tubule epithelium urate secretion is mediated by Mrp4. American Journal of Physiology-Regulatory, Integrative and Comparative Physiology, 295(6), pp.R2024-R2033.

Blankenberg, D., Gordon, A., Von Kuster, G., Coraor, N., Taylor, J., Nekrutenko, A. and Galaxy Team, 2010. Manipulation of FASTQ data with Galaxy. Bioinformatics, 26(14), pp.1783-1785.

Bolger, A.M., Lohse, M. and Usadel, B., 2014. Trimmomatic: a flexible trimmer for Illumina sequence data. Bioinformatics, 30(15), pp.2114-2120.

Borst, P., Evers, R., Kool, M. and Wijnholds, J., 2000. A family of drug transporters: the multidrug resistance-associated proteins. Journal of the National Cancer Institute, 92(16), pp.1295-1302. 
395

396

397

398

399

400

401

402

403

404

405

406

407

408

409

410

411

412

413

414

415

416

417

418

419

420

421

422

423

424

425

426

427

428

Burckhardt, G., 2012. Drug transport by organic anion transporters (OATs). Pharmacology \& therapeutics, 136(1), pp.106-130.

Choudhuri, S. and Klaassen, C.D., 2006. Structure, function, expression, genomic organization, and single nucleotide polymorphisms of human ABCB1 (MDR1), ABCC (MRP), and ABCG2 (BCRP) efflux transporters. International journal of toxicology, 25(4), pp.231-259.

Cole, S.P.C., Bhardwaj, G., Gerlach, J.H., Mackie, J.E., Grant, C.E., Almquist, K.C., Stewart, A.J., Kurz, E.U., Duncan, A.M.Y. and Deeley, R.G., 1992. Overexpression of a transporter gene in a multidrug-resistant human lung cancer cell line. Science, 258(5088), pp.1650-1654.

Cuthbert, R., Pain, D.J., Green, R.E., Swan, G. and Swarup, D., 2007. Comparative toxicity studies of NSAIDs in birds: A criticism of Reddy et al. Anim. Conserv, 9, pp.349-354.

De Castro, E., Sigrist, C.J., Gattiker, A., Bulliard, V., Langendijk-Genevaux, P.S., Gasteiger, E., Bairoch, A. and Hulo, N., 2006. ScanProsite: detection of PROSITE signature matches and ProRule-associated functional and structural residues in proteins. Nucleic acids research, 34(suppl_2), pp. W362-W365.

Edgar, R.C., 2004. MUSCLE: multiple sequence alignment with high accuracy and high throughput. Nucleic acids research, 32(5), pp.1792-1797.

El-Sheikh, A.A., Van Den Heuvel, J.J., Koenderink, J.B. and Russel, F.G., 2007. Interaction of nonsteroidal anti-inflammatory drugs with multidrug resistance protein (MRP) 2/ABCC2-and MRP4/ABCC4-mediated methotrexate transport. Journal of Pharmacology and Experimental Therapeutics, 320(1), pp.229-235.

Felsenstein, J., 1985. Confidence limits on phylogenies: an approach using the bootstrap. Evolution, 39(4), pp.783-791.

Gasteiger, E., Gattiker, A., Hoogland, C., Ivanyi, I., Appel, R.D. and Bairoch, A., 2003. ExPASy: the proteomics server for in-depth protein knowledge and analysis. Nucleic acids research, 31(13), pp.3784-3788.

Grabherr, M.G., Haas, B.J., Yassour, M., Levin, J.Z., Thompson, D.A., Amit, I., Adiconis, X., Fan, L., Raychowdhury, R., Zeng, Q. and Chen, Z., 2011. Full-length transcriptome assembly from RNA-Seq data without a reference genome. Nature biotechnology, 29(7), p.644.

Green, R.E., Newton, I.A.N., Shultz, S., Cunningham, A.A., Gilbert, M., Pain, D.J. and Prakash, V., 2004. Diclofenac poisoning as a cause of vulture population declines across the Indian subcontinent. Journal of Applied ecology, 41(5), pp.793-800.

Haimeur, A.G.R.G.S.P., Conseil, G., Deeley, R.G. and Cole, S.P., 2004. The MRP-related and $\mathrm{BCRP} / \mathrm{ABCG} 2$ multidrug resistance proteins: biology, substrate specificity and regulation. Current drug metabolism, 5(1), pp.21-53. 
429

430

431

432

433

434

435

436

437

438

439

440

441

442

443

444

445

446

447

448

449

450

451

452

453

454

455

456

457

458

459

460

461

Haritova, A.M., Schrickx, J. and Fink-Gremmels, J., 2010. Expression of drug efflux transporters in poultry tissues. Research in veterinary science, 89(1), pp.104-107.

Hipfner, D.R., Deeley, R.G. and Cole, S.P., 1999. Structural, mechanistic and clinical aspects of MRP1. Biochimica et Biophysica Acta (BBA)-Biomembranes, 1461(2), pp.359-376.

Hollenstein, K., Dawson, R.J. and Locher, K.P., 2007. Structure and mechanism of ABC transporter proteins. Current opinion in structural biology, 17(4), pp.412-418.

Jardetzky, O., 1966. Simple allosteric model for membrane pumps. Nature, 211(5052), p.969.

Jarvis, E.D., Mirarab, S., Aberer, A.J., Li, B., Houde, P., Li, C., Ho, S.Y., Faircloth, B.C.,

Nabholz, B., Howard, J.T. and Suh, A., 2014. Whole-genome analyses resolve early branches in the tree of life of modern birds. Science, 346(6215), pp.1320-1331.

Kelley, L.A., Mezulis, S., Yates, C.M., Wass, M.N. and Sternberg, M.J., 2015. The Phyre2 web portal for protein modeling, prediction and analysis. Nature protocols, 10(6), p.845.

Khamdang, S., Takeda, M., Noshiro, R., Narikawa, S., Enomoto, A., Anzai, N., Piyachaturawat, P. and Endou, H., 2002. Interactions of human organic anion transporters and human organic cation transporters with nonsteroidal anti-inflammatory drugs. Journal of Pharmacology and Experimental Therapeutics, 303(2), pp.534-539.

Khare, D., Oldham, M.L., Orelle, C., Davidson, A.L. and Chen, J., 2009. Alternating access in maltose transporter mediated by rigid-body rotations. Molecular cell, 33(4), pp.528-536.

Kiuchi, Y., Suzuki, H., Hirohashi, T., Tyson, C.A. and Sugiyama, Y., 1998. cDNA cloning and inducible expression of human multidrug resistance associated protein 3 (MRP3) 1. FEBS letters, 433(1-2), pp.149-152.

Klein, I., Sarkadi, B. and Váradi, A., 1999. An inventory of the human ABC proteins. Biochimica et Biophysica Acta (BBA)-Biomembranes, 1461(2), pp.237-262.

König, J., Nies, A.T., Cui, Y., Leier, I. and Keppler, D., 1999. Conjugate export pumps of the multidrug resistance protein (MRP) family: localization, substrate specificity, and MRP2mediated drug resistance. Biochimica et Biophysica Acta (BBA)-Biomembranes, 1461(2), pp.377-394.

König, J.Ö.R.G., Nies, A.T., Cui, Y.U.N.H.A.I. and Keppler, D.I.E.T.R.I.C.H., 2003. MRP2, the apical export pump for anionic conjugates (pp. 423-443). Academic Press, London.

Kool, M., de Haas, M., Scheffer, G.L., Scheper, R.J., van Eijk, M.J., Juijn, J.A., Baas, F. and Borst, P., 1997. Analysis of expression of cMOAT (MRP2), MRP3, MRP4, and MRP5, homologues of the multidrug resistance-associated protein gene (MRP1), in human cancer cell lines. Cancer research, 57(16), pp.3537-3547. 
462 Kool, M., Van Der Linden, M., De Haas, M., Baas, F. and Borst, P., 1999. Expression of human 463 MRP6, a homologue of the multidrug resistance protein gene MRP1, in tissues and cancer cells. 464 Cancer Research, 59(1), pp.175-182.

465 Kool, M., Wijnholds, J., Evers, R., Holló, Z., Scheffer, G.L., de Haas, M., van der Linden, M., 466 Mol, C.A.A.M., van Deemter, L., Scheper, R.J. ., Baas, F., Borst, P., 1998. Proc. AACR 39167 467 (Abstract).

468 Krishnamachary, N. and Center, M.S., 1993. The MRP gene associated with a non-P469 glycoprotein multidrug resistance encodes a 190-kDa membrane bound glycoprotein. Cancer 470 Research, 53(16), pp.3658-3661.

471 Kumar, S., Stecher, G., Li, M., Knyaz, C. and Tamura, K., 2018. MEGA X: molecular 472 evolutionary genetics analysis across computing platforms. Molecular biology and 473 evolution, 35(6), pp.1547-1549.

474 Lee, K., Belinsky, M.G., Bell, D.W., Testa, J.R. and Kruh, G.D., 1998. Isolation of MOAT-B, a 475 widely expressed multidrug resistance-associated protein/canalicular multispecific organic anion 476 transporter-related transporter. Cancer research, 58(13), pp.2741-2747.

477 Linton, K.J. and Higgins, C.F., 1998. The Escherichia coli ATP-binding cassette (ABC) proteins. 478 Molecular microbiology, 28(1), pp.5-13.

479 Mirski, S.E., Gerlach, J.H. and Cole, S.P., 1987. Multidrug resistance in a human small cell lung 480 cancer cell line selected in adriamycin. Cancer research, 47(10), pp.2594-2598.

481 Naidoo, V. and Swan, G.E., 2009. Diclofenac toxicity in Gyps vulture is associated with 482 decreased uric acid excretion and not renal portal vasoconstriction. Comparative Biochemistry 483 and Physiology Part C: Toxicology \& Pharmacology, 149(3), pp.269-274.

484 Naidoo, V., Duncan, N., Bekker, L. and Swan, G., 2007. Validating the domestic fowl as a 485 model to investigate the pathophysiology of diclofenac in Gyps vultures. Environmental 486 toxicology and pharmacology, 24(3), pp.260-266.

487 Naidoo, V., Wolter, K., Cuthbert, R. and Duncan, N., 2009. Veterinary diclofenac threatens 488 Africa's endangered vulture species. Regulatory toxicology and pharmacology, 53(3), pp.205489208

490 Nei, M. and Kumar, S., 2000. Molecular evolution and phylogenetics. Oxford university press.

491 Newstead, S., Fowler, P.W., Bilton, P., Carpenter, E.P., Sadler, P.J., Campopiano, D.J., Sansom, 492 M.S. and Iwata, S., 2009. Insights into how nucleotide-binding domains power ABC transport. 493 Structure, 17(9), pp.1213-1222.

494 Nozaki, Y., Kusuhara, H., Kondo, T., Iwaki, M., Shiroyanagi, Y., Nakayama, H., Horita, S., 495 Nakazawa, H., Okano, T. and Sugiyama, Y., 2007. Species difference in the inhibitory effect of 
496

497

498

499

500

501

502

503

504

505

506

507

508

509

510

511

512

513

514

515

516

517

518

519

520

521

522

523

524

525

526

527

nonsteroidal anti-inflammatory drugs on the uptake of methotrexate by human kidney slices. Journal of Pharmacology and Experimental Therapeutics, 322(3), pp.1162-1170.

Oaks, J.L., Gilbert, M., Virani, M.Z., Watson, R.T., Meteyer, C.U., Rideout, B.A., Shivaprasad, H.L., Ahmed, S., Chaudhry, M.J.I., Arshad, M. and Mahmood, S., 2004. Diclofenac residues as the cause of vulture population decline in Pakistan. Nature, 427(6975), p.630.

Oldham, M.L., Davidson, A.L. and Chen, J., 2008. Structural insights into ABC transporter mechanism. Current opinion in structural biology, 18(6), pp.726-733.

Oldham, M.L., Khare, D., Quiocho, F.A., Davidson, A.L. and Chen, J., 2007. Crystal structure of a catalytic intermediate of the maltose transporter. Nature, 450(7169), p.515.

Omasits, U., Ahrens, C.H., Müller, S. and Wollscheid, B., 2013. Protter: interactive protein feature visualization and integration with experimental proteomic data. Bioinformatics, 30(6), pp.884-886.

Punta, M. and Ofran, Y., 2008. The rough guide to in silico function prediction, or how to use sequence and structure information to predict protein function. PLoS Comput Biol, 4(10), p.e1000160.

Rees, D.C., Johnson, E. and Lewinson, O., 2009. ABC transporters: the power to change. Nature reviews Molecular cell biology, 10(3), p.218.

Reid, G., Wielinga, P., Zelcer, N., van der Heijden, I., Kuil, A., de Haas, M., Wijnholds, J. and Borst, P., 2003. The human multidrug resistance protein MRP4 functions as a prostaglandin efflux transporter and is inhibited by nonsteroidal antiinflammatory drugs. Proceedings of the National Academy of Sciences, 100(16), pp.9244-9249.

Ren, X.Q., Furukawa, T., Haraguchi, M., Sumizawa, T., Aoki, S., Kobayashi, M. and Akiyama, S.I., 2004. Function of the ABC signature sequences in the human multidrug resistance protein 1. Molecular pharmacology, 65(6), pp.1536-1542.

Sanger, F. and Coulson, A.R., 1975. A rapid method for determining sequences in DNA by primed synthesis with DNA polymerase. Journal of molecular biology, 94(3), pp.441-448.

Sekine, T., Miyazaki, H. and Endou, H., 2006. Molecular physiology of renal organic anion transporters. American Journal of Physiology-Renal Physiology, 290(2), pp.F251-F261.

Shultz, S., Baral, H.S., Charman, S., Cunningham, A.A., Das, D., Ghalsasi, G.R., Goudar, M.S., Green, R.E., Jones, A., Nighot, P. and Pain, D.J., 2004. Diclofenac poisoning is widespread in declining vulture populations across the Indian subcontinent. Proceedings of the Royal Society of London. Series B: Biological Sciences, 271(supp1_6), pp.S458-S460. 
528 Smith, P.C., Karpowich, N., Millen, L., Moody, J.E., Rosen, J., Thomas, P.J. and Hunt, J.F., 529 2002. ATP binding to the motor domain from an ABC transporter drives formation of a 530 nucleotide sandwich dimer. Molecular cell, 10(1), pp.139-149.

531 Sonnhammer, E.L., Von Heijne, G. and Krogh, A., 1998, July. A hidden Markov model for 532 predicting transmembrane helices in protein sequences. In Ismb (Vol. 6, pp. 175-182).

533 Swan, G.E., Cuthbert, R., Quevedo, M., Green, R.E., Pain, D.J., Bartels, P., Cunningham, A.A., 534 Duncan, N., Meharg, A.A., Lindsay Oaks, J. and Parry-Jones, J., 2006. Toxicity of diclofenac to 535 Gyps vultures. Biology letters, 2(2), pp.279-282.

536 Sweet, D.H., 2005. Organic anion transporter (Slc22a) family members as mediators of toxicity. 537 Toxicology and applied pharmacology, 204(3), pp.198-215.

538 Sweet, D.H., 2010. Renal organic cation and anion transport: From physiology to genes.

539 Tamura, K. and Nei, M., 1993. Estimation of the number of nucleotide substitutions in the 540 control region of mitochondrial DNA in humans and chimpanzees. Molecular biology and 541 evolution, 10(3), pp.512-526.

542 Taniguchi, K., Wada, M., Kohno, K., Nakamura, T., Kawabe, T., Kawakami, M., Kagotani, K., 543 Okumura, K., Akiyama, S.I. and Kuwano, M., 1996. A human canalicular multispecific organic 544 anion transporter (cMOAT) gene is overexpressed in cisplatin-resistant human cancer cell lines 545 with decreased drug accumulation. Cancer Research, 56(18), pp.4124-4129.

546 Van Wert, A.L., Gionfriddo, M.R. and Sweet, D.H., 2010. Organic anion transporters: discovery, 547 pharmacology, regulation and roles in pathophysiology. Biopharmaceutics \& drug disposition, 548 31(1), pp.1-71.

549 Walker, J.E., Saraste, M., Runswick, M.J. and Gay, N.J., 1982. Distantly related sequences in the 550 alpha-and beta-subunits of ATP synthase, myosin, kinases and other ATP-requiring enzymes and 551 a common nucleotide binding fold. The EMBO journal, 1(8), pp.945-951.

552 Ward, A., Reyes, C.L., Yu, J., Roth, C.B. and Chang, G., 2007. Flexibility in the ABC 553 transporter MsbA: Alternating access with a twist. Proceedings of the National Academy of 554 Sciences, 104(48), pp.19005-19010.

555 Zhang, G., Li, C., Li, Q., Li, B., Larkin, D.M., Lee, C., Storz, J.F., Antunes, A., Greenwold, 556 M.J., Meredith, R.W. and Ödeen, A., 2014. Comparative genomics reveals insights into avian 557 genome evolution and adaptation. Science, 346(6215), pp.1311-1320.

558 Zhang, P., Tian, X., Chandra, P. and Brouwer, K.L., 2005. Role of glycosylation in trafficking of 559 Mrp2 in sandwich-cultured rat hepatocytes. Molecular pharmacology, 67(4), pp.1334-1341. 
Figure 1

MRP2 phylogenetic tree

Reconstruction of phylogenetic relationship between MRP2 genes of different avian species by maximum likelihood best model: bootstrap.

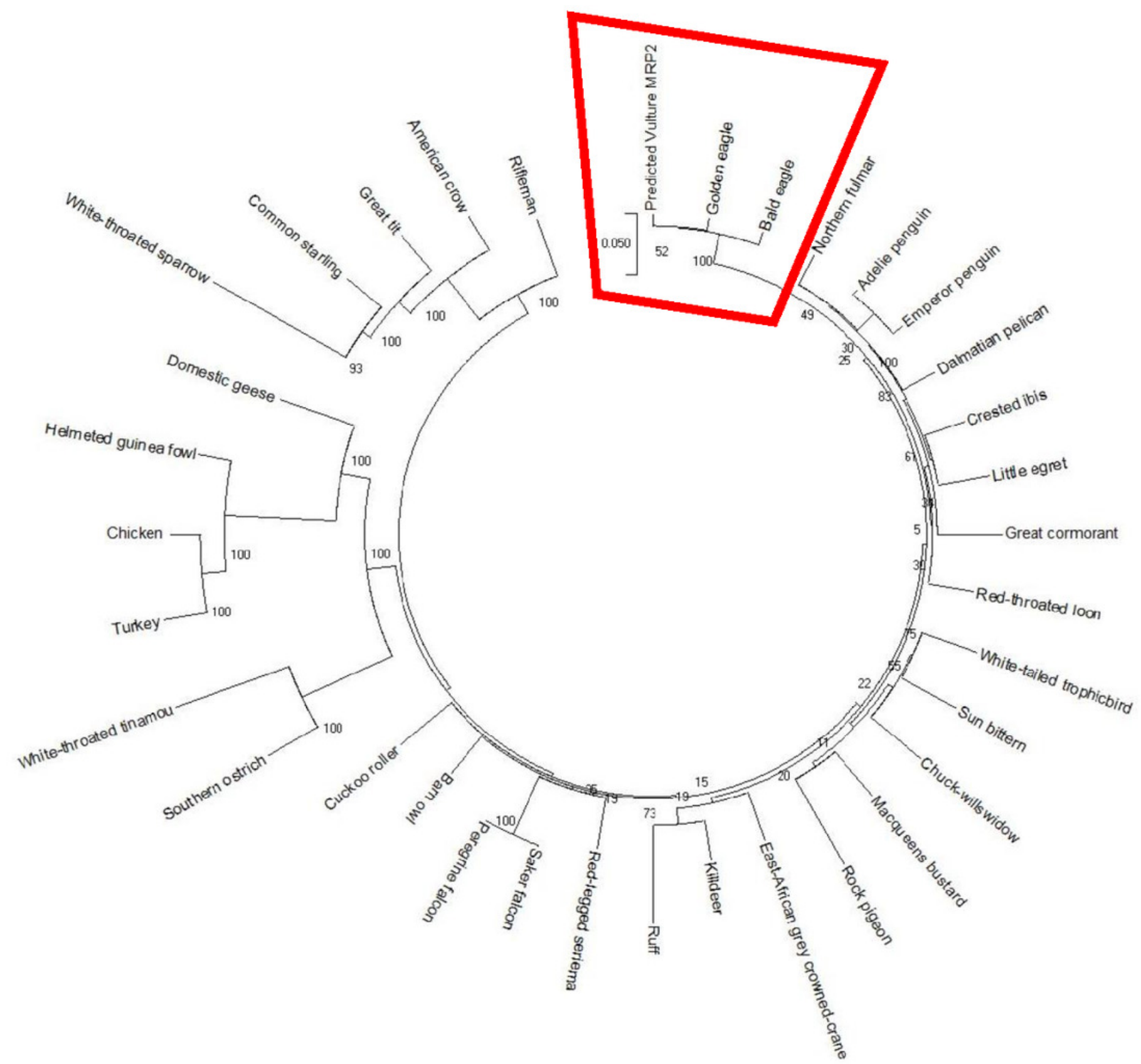


Figure 2

MRP4 phylogenetic tree

Reconstruction of phylogenetic relationship between MRP4 genes of different avian species by maximum likelihood best model: bootstrap. 


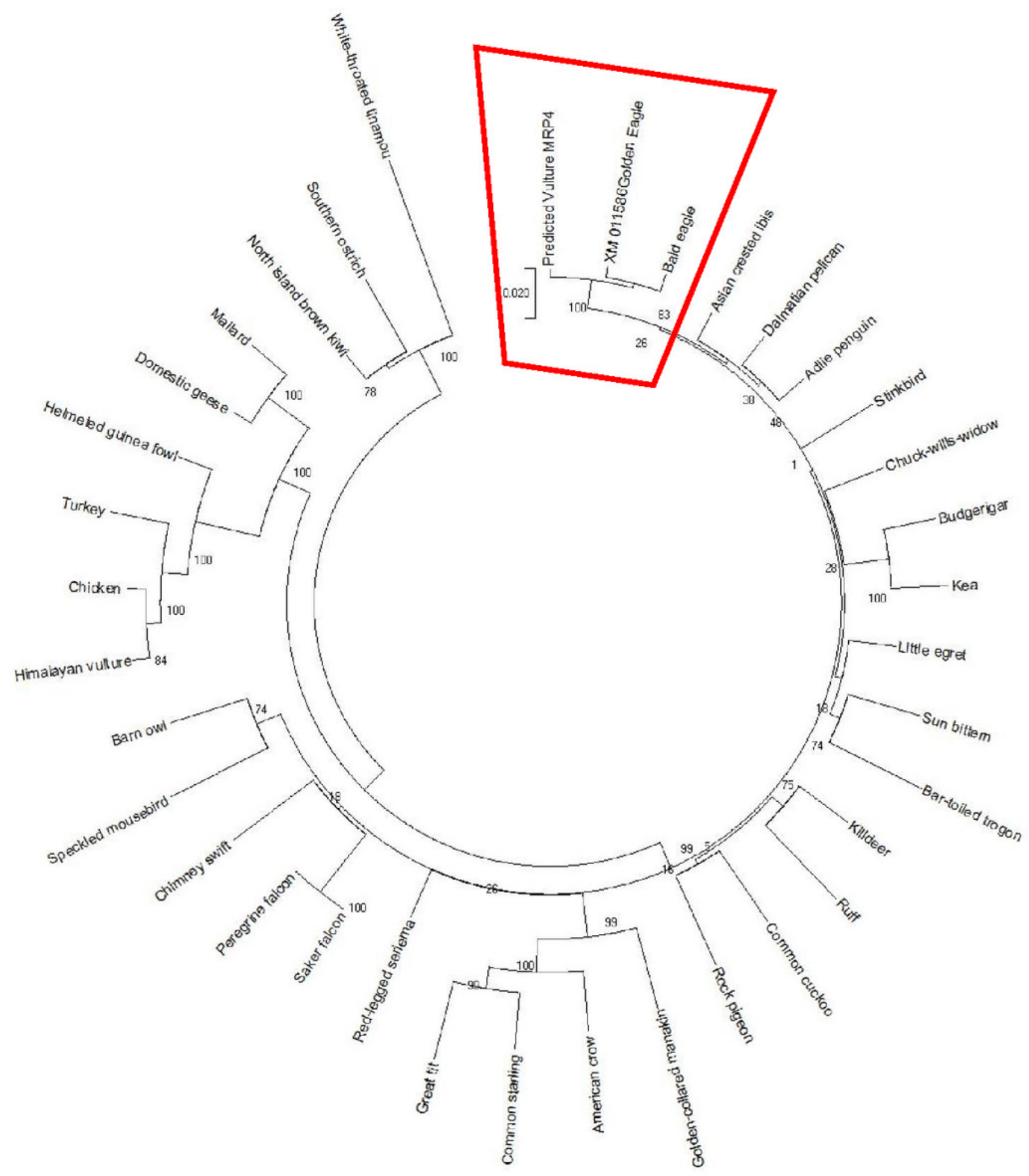




\section{Figure 3}

Presentation of transmembrane helices with $\mathrm{N}$-gyclan motif sites and 3-D structures.

Pictorial presentation of transmembrane helices and N-gyclan motif sites from A) MRP2 containing $13 \mathrm{~N}$-glycan motif sites with $16 \mathrm{TMH}$ and B) MRP4 comprising of $5 \mathrm{~N}$-glycan motif sites with 11 TMH. Also shown are the corresponding 3-D structures of MRP2 and MRP4, illustrated by the PHYRE2 software, showing the presence of two NDBs. 

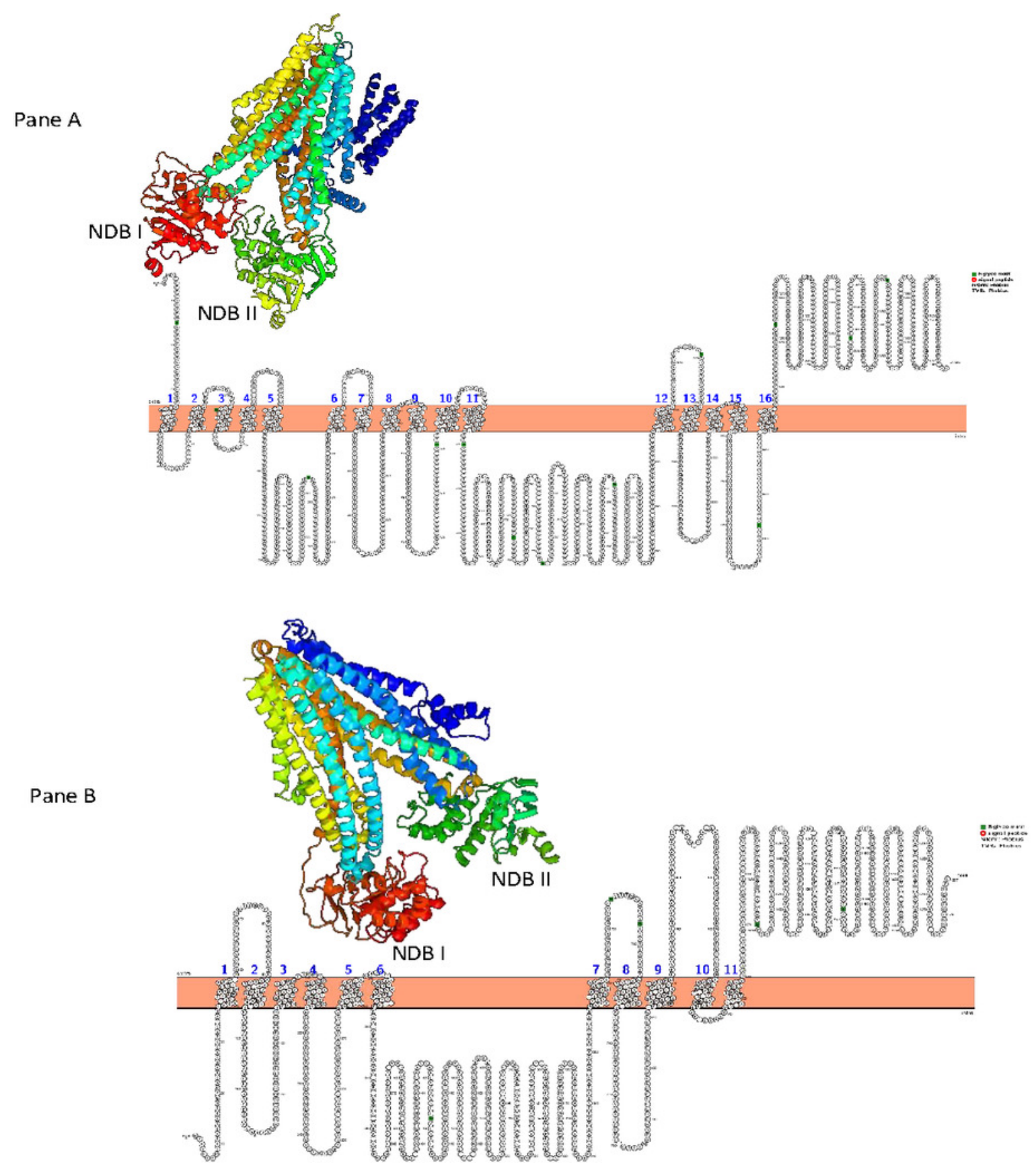

MRP4

MRP2

Pane B 
Figure 4

Walker sequence and $A B C$ signature motif

comparison of the Walker A, ABC Signature motif and Walker B sequence of the first

nucleotide binding domain of the vulture MRP2 and MRP4 in comparison on human MRP (The protein sequences used with their GenBank accession numbers were MRP2-NP_000383.2, MRP3-AAD02845.1,MRP4NP_005836.2, MRP5-NP_005679.2 


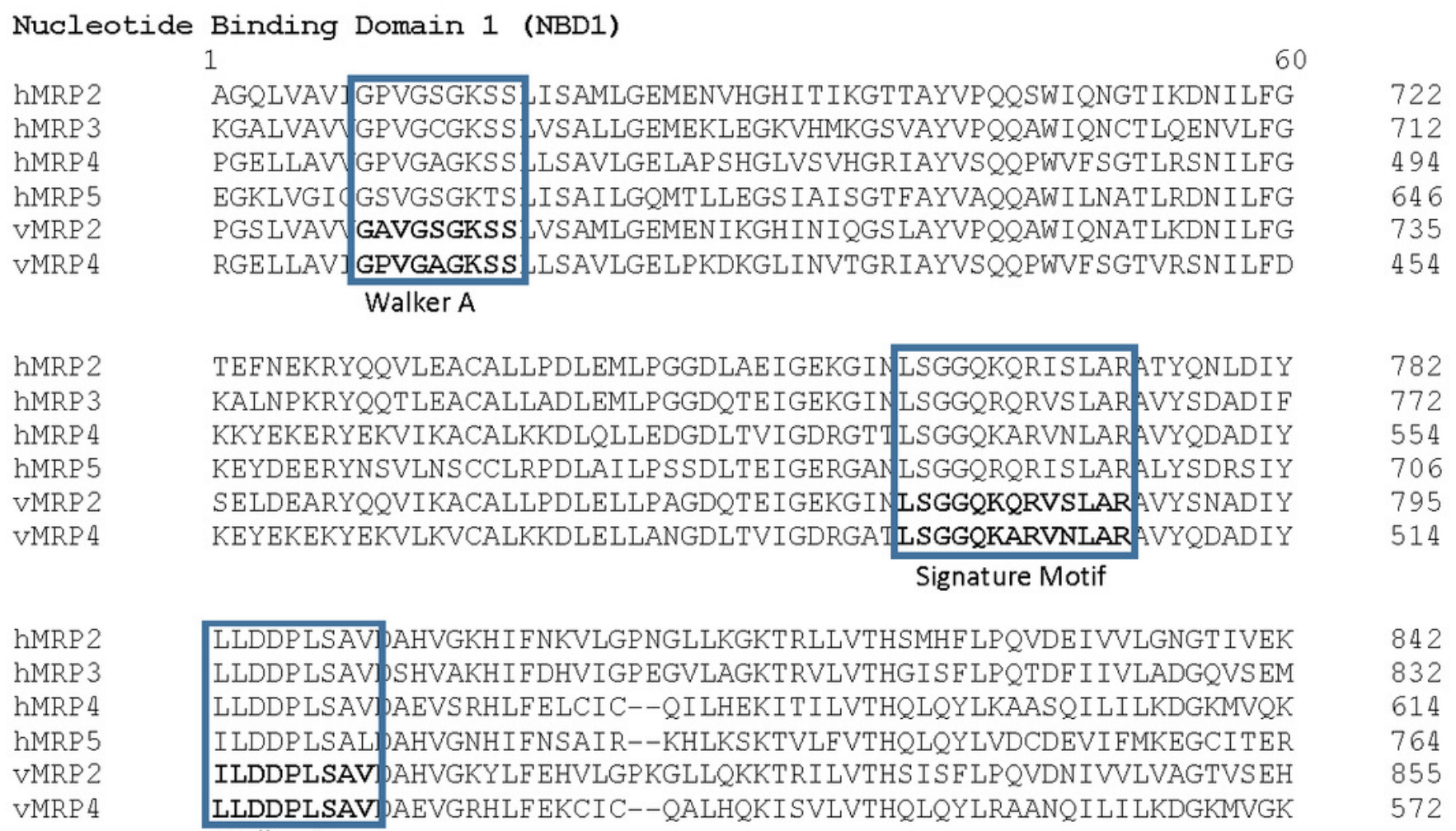

\section{Nucleotide Binding Domain 2 (NBD2)}

hMRP2 FNNYQVRYRPELDLVLRGITCDIGSMEKIGVVGR? GAGKSSLTICLFRI LEAAGGQI I ID hMRP3 hMRP 4 hMRP5 VMRP2 VMRP 4 FRNYSVRYRPGLDLVLRDLS LHVHGGEKVGIVGR? FDNVNFMYSPGGP LVLKHLTALIKSQEKVGIVGRTGA GAGKSSMT CLFRILEAAKGEIRID FENAEMRYRENLP LVLKKVSFT IKPKEKIGIVGR? GSGKSS LGI LALFRLVELSGGCIKID FVDYKVRYRP ELELVLQGI TCN IGSTEKVGVVGR GAGKSSLTICLERVLEAAGGT I I ID FENVNFTYS LDGP LVLRHLSVLIKPKEKVGIVGRT GAGKSSLI. ALFRLAEP-EGRIWID Walker A

hMRP2 LQLGLSHEVTEAGGN LS IGQRQLLCLGR ALLRKSKI IVLDEAT AAVDLETDNLIQTTIQN QPAGLDFQCSEGGEN LSVGQRQLVCLARALLRKSRI IVLDEAT AAIDLETDNLIQAT I RT LPGKMDTELAESGSN FSVGQRQLVCLAR A LRKNQI I I DEAT ANVDP RTDELIQKKIRE ILDEAT AAMDTETDLLIQETIRE LPEGLLHLVSEAGEN LSVGQRQLVCLAR ALLRKAKI ILDEAT AAVDLETDHLIQTT I RS VMRP 4 LPNKMEMQLAESGSN FSVGQRQLVCLARAVLKKNRI I I Signature Motif I IDEAT ANVDPRTDEF IQKT IRE 